\title{
MOBILIZAÇÃO NEURODINÂMICA E REGENERAÇÃO NERVOSA PERIFÉRICA: REVISÃO BIBLIOGRÁFICA
}

\author{
Emyle Martins Lima Programa de Pós-graduação em Medicina e Saúde, \\ Faculdade de Medicina, Universidade Federal da \\ Bahia. Laboratório de Eletroestimulação \\ Funcional, Departamento de Biomorfologia, \\ Universidade Federal da Bahia.
}

Cavalcante, D. M. Programa de Pós-graduação em Medicina e Saúde, Faculdade de Medicina, Universidade Federal da Bahia. Laboratório de Eletroestimulação Funcional, Departamento de Biomorfologia, Universidade Federal da Bahia.

Iasmyn Adelia Victor Fernandes
de Oliveira

Eulália Silva dos Santos Pinheiro
Laboratório de Eletroestimulação Funcional, Departamento de Biomorfologia, Universidade Federal da Bahia.

Programa de Pós-graduação em Medicina e Saúde, Faculdade de Medicina, Universidade Federal da Bahia. Laboratório de Eletroestimulação Funcional, Departamento de Biomorfologia, Universidade Federal da Bahia.

Ana Maria Blanco Martinez

Laboratório de Neuroregeneração e Reparo, Universidade Federal do Rio de Janeiro.

Abrahão Fontes Baptista
Programa de Pós-graduação em Medicina e Saúde, Faculdade de Medicina, Universidade Federal da Bahia. Centro de Pesquisas Gonçalo Moniz Fundação Oswaldo Cruz (FIOCRUZ). Laboratório de Eletroestimulação Funcional, Departamento de Biomorfologia, Universidade Federal da Bahia.

\begin{abstract}
Resumo
Introdução: A mobilização neurodinâmicaé indicada em uma série de condições que afetam o nervo periférico, mas pouco se conhece sobre seus efeitos regenerativos.Objetivos: Descrever como a mobilização neurodinâmica pode interferir nas propriedades fisiológicas e mecânicas do nervo.Métodos: Esta revisão narrativa incluiu artigos registrados entre 1973 e 2012 no PubMed, escritos na língua Portuguesa e Inglesa e livros envolvendo estes tópicos. Discussão e Conclusão: Após uma lesão nervosa periférica uma série de eventos podem influenciar a regeneração, incluindo transporte axonal e movimento de fluidos, a carga mecânica aplicada no tecido neural e no tipo de lesão. Estes aspectos podem ser influenciados pela mobilização neurodinâmica. A dose, incluindo a magnitude da carga, velocidade e tempo são fatores que devem ser modulados para uma resposta ótima, enquanto a tensão, imobilidade ou hipomobilidade, podem ter efeitos negativos sobre a recuperação.
\end{abstract}

Palavras-chave: Esmagamento do nervo; Reabilitação; Nervo ciático;Neurodinâmica; Técnicas de fisioterapia.

\section{NEURODYNAMIC MOBILIZATION AND PERIPHERAL NERVE REGENERATION: LITERATURE REVIEW}

\footnotetext{
Abstract

Introduction: The neurodynamicmobilization may be indicated in a number of conditions affecting the peripheral nerve, but little is known about its regenerative effects. Objectives: Describe how the neurodynamicmobilization may interfere with the mechanical and physiological properties of the peripheral 
nerve. Methods: This narrative review included all articles registered form 1973 to 2012in PubMed, written in Portuguese and English languages, and books involving this topic. Discussion and conclusion: After a peripheral nerve injury a series of events can influence regeneration, including the axonal transport and movement of fluids, the mechanical loads applied to the neural tissue and the type of injury. These aspects may be influenced by neurodynamic mobilizations. The dose, including the magnitude of the load, speed and time are factors that tension should be adjusted for optimum response, since both overload, and immobility or hypomobility may have negative effects on the recovery.

Keywords: Nerve crush; Rehabilitation; Sciatic nerve;Neurodynamic; Physical therapy techniques;Rats.

\section{INTRODUÇÃO}

Os nervos periféricos estão sujeitos a diversos tipos de lesões causadas por processos inflamatórios, auto-imunes e provenientes de traumatismos ou eventos isquêmicos. ${ }^{(1)}$ Após uma lesão, o segmento proximal mantém continuidade com o corpo celular e apresenta atividade regenerativa. Lesões que implicam na perda da continuidade axonal resultam em Degeneração Walleriana (DW) do coto distal à lesão. ${ }^{(2)}$

O sistema nervoso periférico é suprido por uma vasculatura que possui um sistema colateral desenvolvido e que quando traumatizado, conserva um arranjo adequado para manter um fluxo ininterrupto. ${ }^{(3)} \mathrm{O}$ suprimento de energia fornecido pelo sangue interfere no transporte axoplasmático,que participa da manutenção e nutrição dos componentes celulares do axônio. ${ }^{(4)}$

Compressões e alongamentos podem comprometer o fluxo sanguíneo e o transporte axonal ${ }^{(5)}$ através da redução da área de secção transversal e conseqüente aumento da pressão intrafascicular. ${ }^{(3,5,6)}$ Estes efeitos podem alterar a capacidade regenerativa do nervo. ${ }^{(4)}$

Lesões por estiramento agudo ou compressão neural crônica ${ }^{(7)}$ podem alterar as propriedades mecânicas e fisiológicas de umnervo, afetando sua neurodinâmica. ${ }^{(8)}$ Graças ao comportamento visco-elástico ${ }^{(6)}$ do tecido nervoso, suas propriedades estruturais e biomecânicas podem ser modificadas em resposta a estressese/ouposturas. ${ }^{(5)}$ As alterações funcionais que ocorrem no tecido nervoso decorrentes de uma deformação (tensão ou estiramento) dependem do tempo de aplicação, da magnitude a das características da força a qual o nervo é submetido. ${ }^{(3)}$

A mobilização neurodinâmica pode ser aplicada através de movimentos que causam tensionamento ou deslizamento do nervo, para ativar funções fisiológicas e viscoelásticas. É aplicada aos tecidos neurais por aumento da distância entre a extremidade do trato nervoso, através do movimento articular. As manobras têm sido usadas para tratar sintomas de origem neuropática. ${ }^{(1)}$ Sua influência parece ser através do estímulo ao retorno da circulação normal 
no nervo e o incentivo ao transporte axoplasmático. Estes efeitos poderiam ser benéficos no processo de regeneração nervosa periférica, mas não existem estudos que comprovem os efeitos destas manobras para este fim. A parametrização desta técnica é de grande importância, pois na prática clínica fisioterapêutica existe pouca evidência para a aplicação dos diversos métodos utilizados para o tratamento das lesões nervosas periféricas, sejam eles para reduzir queixas álgicas, estimular a condução nervosa ou promover a recuperação funcional. Neste trabalho levantamos aspectos básicos que podem influenciar o efeito da mobilização neurodinâmica quando aplicada a nervos periféricos lesionados.

\section{LESÃO E REGENERAÇÃO NERVOSA PERIFÉRICA}

Após uma lesão nervosa periférica ocorrem alterações morfológicas e funcionais, causando desnervação dos órgãos periféricos. As fibras seccionadas distalmente à lesão sofrem DW. O coto proximal, que continua ligado ao corpo celular, favorece o crescimento das fibras nervosas em direção ao órgão-alvo. Concomitantemente, ocorre uma reação retrógrada, composta por um conjunto de alterações moleculares e celulares na soma dos neurônios axotomizados. ${ }^{(2)}$

No estágio inicial da DW ocorre acúmulo de organelas citoplasmáticas distalmente à lesão, provavelmente decorrente de alterações no transporte axonal e edema endoneural. Neste estágio a condução elétrica está normal e a duração desta fase varia de acordo com o comprimento do coto distal, natureza do nervo e local da lesão. Nesta fase, praticamente não existem alterações no axônio lesado. ${ }^{(3)}$

Em torno de 24 a 48 horas se inicia a desintegração do citoesqueleto e degradação do axoplasma e axolema na região distal ao local da lesão, ${ }^{(2)}$ mediada por aumento nas concentrações intracelulares de cálcio proveniente de depósitos intracelulares ou do espaço extracelular. ${ }^{(3)}$ Este influxo de cálcio para dentro da célula provoca "correntes elétricas de lesão" através do aumento dos potenciais elétricos locais. ${ }^{(4)}$ Neste período, as células de Schwann fragmentam a mielina e fagocitam pequenos restos celulares formando as Bandas de Büngner(BB). As extremidades distais dos neurônios lesionados formam os "cones de crescimento", a partir dos quais são gerados brotos regenerativos, que crescem em direção ao órgão-alvo, guiados pelas BB.

Entre o segundo e o sétimo dia após a lesão, os macrófagos infiltram e penetram na região distal ao local da lesão, fagocitando os fragmentos de mielina. As CS diminuem a 
expressão de proteínas formadoras de mielina e se desdiferenciam, adquirem um fenótipo de pré/não-mielínica e formam as $\mathrm{BB}$, que estimularão o crescimento do neurito em direção à célula-alvo. Os fatores TGF- $\beta 1$ e $\beta 2$ e LIF são sobre-regulados após lesões axonais e estão intimamente ligados ao processo de regeneração no $\mathrm{SNP}^{(5)}$. O TGF- $\beta 1$ associado ao AMPc regula a expressão da proteína GAP-43 ${ }^{(6)}$ que está relacionada com a capacidade da célula lesada em avaliar o ambiente onde ela está se expandindo, amplificando os sinais gerados pelas pistas que orientam o caminho a ser seguido pelo cone de crescimento . O TGF- $\beta 1$ exerce papel de sinalizador para que as CS passem do estado proliferativo/mielinizante, para o estado não-mielinizante necessário para o início do processo de regeneração. ${ }^{(7)}$

A mobilização passiva ou tensionamentos aumentam síntese de proteína muscular, diminuem a degradação de proteína na inervação muscular, aumentam a tensão muscular e estimulam a reinnervação. ${ }^{(8,9)}$ Por isso, hipotetiza-se que a mobilização neural, uma forma de mobilização passiva que se baseia no comportamento mecânico do sistema nervoso, possa influenciar a recuperação funcional após uma lesão nervosa periférica.

\section{TIPOS DE LESÃO}

As lesões nervosas podem ser classificadas dependendo das alterações estruturais e funcionais das fibras nervosas, ${ }^{(9)}$ mais especificamente do déficit da condução nervosa e perda funcional, tais como paralisia motora e alteração sensitiva. ${ }^{(10)}$

Seddon, em 1943 e 1972, propôs uma classificação que descreveu três estágios diferentes da lesão de nervos. No primeiro grau de lesão há continuidade dos axônios, com bloqueio de condução local, e esta lesão corresponde às lesões por compressão, sendo a Neuropraxia o termo mais adequado.

O segundo grau de lesão, conhecido por axoniotmese, implica na perda da continuidade axonal no nível da lesão, porém com preservação dos tubos endoneurais. Corresponde ao avanço da lesão por compressão ou tração, com interrupção da continuidade axonal, resultando em DW do coto distal do nervo, levando a paralisia motora, alterações sensitivas e autonômicas. A estrutura de apoioé fornecida pelaresistência mecânica dostubosendoneuraisem relação comaxônios. ${ }^{(9)}$ A alta força compressivapode romper facilmente a continuidade dos axonios, enquanto os tubos endoneurais permanecem intactos. O prognóstico desta lesão depende do tempo de lesão. Neste caso, como há manutenção das 
Bandas de Büngner, a orientação para o crescimentoaxonal é mantida eentão o prognóstico parareinervação correta dosalvos é bom, correspondendo a uma boa recuperação funcional. ${ }^{(11)}$

Alesão mais grave corresponde a neurotmeseque envolve a perda da continuidade de alguns ou todos os elementos do tronco nervoso, incluindo tubos endoneurais, perineuro e epineuro. Este termo foi usado para descrever uma completa desorganização do nervo, com repercussões severas. ${ }^{(12)}$ Nesta lesão, embora seja possível a regeneração axonal, existe baixa especificidade na reinervação dos órgãos alvo. Axônios motores eferentes quando direcionados para axônios sensoriais aferentes conduzem a degradação ou perda permanente da função. ${ }^{(13,14)}$

\section{TRANSPORTE AXONAL X REGENERAÇÃO NERVOSA PERIFÉRICA}

Lesões de nervos periféricos provocam uma cascata de respostas que são necessárias para uma boa resposta regenerativa. Axônios lesionados devem sinalizar retrogradamente para seus corpos celulares ativarem mecanismos intrínsecos de crescimento e, em seguida, superarem as barreiras físicas e sinais inibitórios no ambiente extracelular para alcançarem os órgãos alvo corretos e promoverem a regeneração funcional. ${ }^{(15)}$

O fluxo axoplasmático torna-se lento, alterando o transporte axonal (anterógrado e retrógado), afetando então a manutenção do citoesqueleto, o controle interno do neurônio e a capacidade regenerativa do nervo. ${ }^{(10)} \mathrm{O}$ transporte axonal movimenta estruturas subcelulares e proteínas do corpo celular para os terminais do axônio e vice-versa, sendo esta comunicação imprescindível para regeneração. Existem descritos atualmente três tipos de transporte axonal: rápido (anterógrado e retrógrado), lento (anterógrado) e mitocondrial. ${ }^{(16)}$

Os componentes sinápticos do caminho dos microtúbulos, as organelas da membrana e os movimentos das mitocôndrias são direcionados do corpo para os teminais axônicos pelo transporte axonal anterógrado rápido e mediado pela proteína motora conhecida como cinesina ${ }^{(17,18)} \mathrm{O}$ componenteretrógrado facilita a reciclagemda membrana e fornece uma via retrógradapara a transmissão deinformações através dotransporte dereceptoresassociadosàs vesículasda membrana. ${ }^{(17)}$ Adineína, presente no axolema, pode mover microtúbulosin vitro a uma velocidade de 14 micrometro/seg. Em comparação as cinesinas, mais rápidas, podem mover seus microtúbulos a aproximadamente 2 a 3 micrometros/seg. ${ }^{(18)}$ 


\section{ANATOMIA DOS NERVOS PERIFÉRICOS}

A anatomia do sistema vascular das raízes nervosas, dispõem-se de tal forma, que nos permite adotar posturas estáticas e dinâmicas, mantendo a função normal do nervo e o sistema ininterrupto. $^{(4)} \mathrm{O}$ sistema nervoso periférico apresenta adaptações compensatórias que permitem o movimento. A morfologia da fibra nervosa, com ondulações tortuosas e capacidade de deslizamentos ${ }^{(19)}$ torna possível a excursão entre perineuro e epineuro. ${ }^{(1)}$

O sistema circulatório também tem uma anatomia própria para permitir a função do nervo. Há uma integração de dois sistemas microvasculares com funções independentes. $\mathrm{O}$ sistema intrínseco, oriundo dos vasos locais, dividi-se e forma anastomose com o sistema intraneural ao alcançar o epineuro. Este sistema é formado peloepineuro, perineuro, plexosendoneuraise seusvasos comunicantes ${ }^{(20)}$ e possui fluxo sanquíneo reversível e dependente de um sistema colateral. ${ }^{(10,21)}$ No plexo epineural há um grande número de arteríolas e vênulas, que correm longitudinalmente, formando numerosas anastomoses, permitindo comunicação com perineuro e endoneuro. Esta capacidade desenvolvida do plexo perineurial tem relação estreita com o leito vascular intrafascicular endoneural, que se estende ao longo de todo o nervo. ${ }^{(20)} \mathrm{O}$ sistema extrínseco, originado de artérias e veias adjacentes, geralmente tem uma aparência tortuosa, permitindo grande comprimento e dessa forma grande variação de amplitude do nervo. ${ }^{(20)}$

\section{EFEITOS DA COMPRESSÃO E TENSÃO NO NERVO PERIFÉRICO}

Emboraexistam poucos estudos que abordem a patomecânica do nervo, sabe-se que os alongamentos e compressões podem reforçar as fases do processo inflamatório decorrente da lesão, acentuando a pressão intrafascicular, com fechamento dos vasos que cruzam o perineuro. ${ }^{(10)}$ Estudos que corroboram com estes achados relatam que após estiramento, ocorrem mudanças na periferia do nervo incluindo alterações no tecido conjuntivo, especialmente o perineuro e epineuro, bem como mudanças na estrutura axonal e mielínica. ${ }^{(22)}$

Nas síndromes de compressão nervosa, a ocorrência de restrição no deslizamento dos nervos periféricos impõe tensão ao nervo durante alguns movimentos extremos e pode ser avaliada clinicamente por testes neurodinâmicos. ${ }^{(23)}$ Estas restrições podem ser atribuídas às alterações fisiopatológicas do tecido conjuntivo do nervo, que têm sido consideradas responsáveis pela mudança no fluxo sanguíneo e isquemia nas fibras nervosas, além de 
diminuir a capacidade de deslizamento e aumentar a tensão do nervo durante movimentos dos membros. ${ }^{(24)}$

Estudos demonstraram que alongamentos no nervo de $8 \%$ e $15 \%$ causam decréscimo no fluxo sanguíneo neural de $50 \%$ e $80 \%$, respectivamente, ${ }^{(25,26)}$ porém, uma tensão de $8,8 \%$ não foi capaz de reduzir velocidade de condução, enquanto $16,1 \%$ diminui a velocidade de pico abaixo $66 \%$ de valor basal. ${ }^{(27)}$ Tensões provocadas por estiramento entre $10 \%$ a $12 \%$ do comprimento de repouso por uma hora levaram a bloqueio completo de condução, ${ }^{(28)} \mathrm{com}$ deterioração da função do nervo após 30 a 90 minutos. ${ }^{(29)}$

Quando o nervo está inflamado, um aumento mínimo de $3 \%$ em sua tensão é suficiente para gerar impulsos ectópicos, desencadeando alguns sintomas. Entretanto, o restabelecimento do fluxo sanguíneo coincide com a recuperação da função. ${ }^{(30)}$ Estudos demonstram que compressões e alongamentos estão associados com efeitos diretos sobre o nervo. ${ }^{(31)}$

\section{MOBILIZAÇÃo NEURODINÂMICA E LESÃO NERVOSA PERIFÉRICA}

Tensão Neural era o termo utilizado no passado para descrever disfunção do sistema nervoso periférico. Mais recentemente foram incluídos conceitos tais como estrutura e função do sistema nervoso e o termo mais comumente aceito é neurodinâmica. ${ }^{(1)}$ Os testes neurodinâmicostêm sido utilizados para avaliar a sensibilidade e deduzir patologias mecânicas dos nervos periféricos, destacando-se a elevação da perna estendida (EPE), um teste clínico confiável para indicar disfunções lombares visto que causa excursão e aumento da tensão na raiz nervosa. ${ }^{(32)}$

A mobilização neurodinâmica envolve exercícios que podem ser classificados como deslizantes ou tensionantes. Os exercícios deslizantes movimentam duas articulações simultaneamente, de tal forma que enquanto uma alonga o leito nervoso, a tensão é contrabalançada por um movimento da articulação adjacente, por exemplo, na extensão do punho associada à flexão de cotovelo. ${ }^{(33)}$ Os benefícios de tal técnica incluem facilitação do deslizamento do nervo, redução da sua aderência sobre tecidos adjacentes, dispersão de substâncias fluídas nocivas, aumento da vascularização neural e melhora do fluxo axoplasmático. $^{(1)}$

Os Exercícios tensionantes são aqueles em que apenas uma articulação é movida causando alongamento neural e das estruturas adjacentes. ${ }^{(32)}$ Os resultados a respeito da 
aplicação deste tipo de exercício são controversos. Os efeitos deletérios já foram demonstrados em estudos que aplicam tensão sobre nervos lesionados experimentalmente. ${ }^{(33)}$

O primeiro estudo a mostrar que movimentos amplos impedem a recuperação funcional de forma significativa, principalmente pelo aumento do tecido conectivo endoneural (colágeno) e redução da angiogênese neural no segmento do nervo suturado, apresenta a redução da tensão no local do reparo da transecção como princípio fundamental. Dados deste estudo sugerem que movimento do nervo no local da sutura aumenta significativamente o tecido conectivo endoneural, observado na avaliação do $5^{\circ}$ dia após cirurgia, porém em 3 semanas os resultados não são expressivos. ${ }^{(34)}$

Em um trabalho em quese investigou tensões crescentes classificadas como baixa, moderada e alta tensão após lesão por transecção, apenas a alta tensão foi capaz de gerar efeitos adversos, com comprometimento da função locomotora e alteração dos aspectos morfológicos do nervo. ${ }^{(35)}$ Cargas locais abaixo de $0,39 \mathrm{~N}$ não foram prejudiciais, mas cargas superiores podem aumentar a tensão e a pressão intraneural, ${ }^{(36)}$ desencadear descargas ectópicas e exacerbar outros sintomas. ${ }^{(30)}$

Por outro lado, o estiramento neural pode reduzir o edema e comprometimento circulatório intraneural e induzir a irrigação do nervo devido ao efeito de bombeamento provocado pelo exercício. ${ }^{(37)} \mathrm{O}$ conhecimento sobre a mecânica do nervo pode indicar a articulação ideal a se movimentar na escolha de um tratamento ${ }^{(38)}$ e a escolha correta pode ser útil em condições clínicas como a síndrome do túnel do carpo (STC). ${ }^{(33)}$ A velocidade com que se aplica a técnica também é crítica, mantendo uma relação direta com a tensão no nervo. O aumento da tensão secundário ao aumento da velocidade de mobilização provoca déficit na velocidade de condução nervosa e no transporte axonal. ${ }^{(39)} \mathrm{O}$ local onde a mobilização é aplicada também pode influenciar a resposta ao tratamento, já que os membros superiores estão mais sujeitos a efeitos benéficos do que os membros inferiores. ${ }^{(40)}$

\section{CONCLUSÃO}

Após uma lesão nervosa periférica, uma série de eventos pode influenciar a regeneração funcional, incluindo aspectos que envolvem o funcionamento mecânico do nervo. O tipo de lesão é fator critico, com as axoniotmeses tendo melhor prognóstico do que as neurotmeses. O transporte axonal e a circulação de fluidos no nervo são determinantes para o sucesso da regeneração e ambos podemser influenciados por aplicações de cargas mecânicas 
sobre o tecido neural. A dose, incluindo a magnitude da carga, a velocidade e o tempo de tensionamento são fatores que devem ser modulados para uma resposta ótima, já que tanto o excesso de tensão, quanto a hipomobilidade ou imobilidade podem ter efeitos negativos sobre a recuperação. Embora existam várias evidências envolvendo o tensionamento de nervos periféricos após lesão,estudos futuros devem abordar especificamente a mobilização neurodinâmica, pois suas características fundamentais diferem de tensões aplicadas com movimentos inespecíficos sobre o tronco e os membros.

\section{REFERÊNCIAS}

1. Shacklock M. Neurodinâmicageral. In: Elsevier BH-, editor. Neurodinâmicaclínica: uma nova abordagem do tratamento da dor e da disfunção musculo esquelética. Rio de Janeiro; 2006. p. 16.

2. Stoll G, Müller HW. Nerve injury, axonal degeneration and neural regeneration: basic insights. Brain Pathology. 1999;9:313-25.

3. Allodi, I., Udina E., and Navarro, X.Specificity of peripheral nerve regeneration: interactions at the axon level. ProgNeurobiol 2012;98:16-37.

4. MartinezAM, RibeiroLC.Ultrasturctural localization of calcium in peripheral nerve fibers undergoing Wallerian-Degeneration: an oxalate-pyroantimoniate and X-ray microanalysis study. J SubmicroscCytolPathol.1998;30:451-8.

5. McCaig CDR, RajnicekAM, SONG B, Zhao M. Has electric growth cone guidance found its potential? Trends in Neurosciences. 2002;25(7):354-9.

6. Stoll G, Müller HW. Nerve injury, axonal degeneration and neural regeneration: basic insights. Brain Pathology. 1999;9:313-25.

7. Stewart HJC, Curtis R,Jessen, KR,Mirsky R. TGF-beta and cAMP regulate GAP-43 expression in Schwann cells and reveal the association of this protein with the trans-Golgi network. JNeurosci. 1995;7(8):1761-72.

8. Fu SY, Gordon T.The cellular and molecular basis of peripheral nerve regeneration. . MolNeurobiol. 1997;14(12):67-116.

9. SqueccoR,Kerm H, Biral D, Rossini K, FranciniF.Mechano-sensitivity of normal and long term denervated soleus muscle of the rat. Neurol Res. 2008;30(2):155-9.

10. GoranLundborg. Acute nerve compression. In: Elsevier, editor. Nerve Injury and Repair: Rgeneration, Reconstruction, and Cortical Remodeling. Second Edition ed. USA1988.

11. Butler DS. Mobilização do Sistema Nervoso. São Paulo: Manolle; 2003. 
12. Valero-Cabré A, Navarro X. Functional impact of axonal misdirection after peripheral nerve injuries followed by graft or tube repair. J Neurotrauma. 2002;19(11):147585.;19(11):1475-85.

13. GoranLundborg. L. Nerve Injury and Repair: Regeneration, Reconstruction and Cortical. 2nd ed. 1988.

14. GoranLundborg, Richard P. Nerve injury and repair - a challenge to the plastic brain. J PeripherNerv Syst. 2003;8(4):209-26.

15. Michaelevski I. Axonal Transport Proteomics Reveals Mobilization of Translation Machinery to the Lesion Site in Injured Sciatic Nerve, Molecular \& Cellular Proteomics. The American Society for Biochemistry and Molecular Biology. 2010.

16. Hammond C. Cellular and Molecular Neurobiology. $2^{\text {nd }}$ ed. Academic Press EUA: Califórnia; 2001. p. 481.

17. Morris JB, Davies WR. Molecular Biology of the Neuron. $2^{\text {nd }}$ ed. EUA: New York; 2004. $499 \mathrm{p}$.

18. AlbertsB. Molecular Biology of the Cell. $5^{\text {a }}$ ed 1999.

19. Walsh MT. Upper limb neural tension testing and mobilization. Fact, fiction, and a practical approach. Journal of hand therapy: official journal of the American Society of Hand Therapists. 2005;18(2):241-58. Epub 2005/05/14.

20. GoranLundborg, Goteborg Swwden. structure and function or the intraneuralmicrovessels as related to trauma, edema formation, and nerve function. J Bone Joint Surg Am 1975;57:938 -48.

21. Richard FE, Wayne AH, Peter JM. Comparison of Longitudinal Sciatic Nerve Movement withDiferent Mobilization Exercises: An In Vivo Study Utilizing Ultrasound Imaging. J Orthop Sports PhysTher 2012. 2012;42(8):667-75.

22. Rydevik BL, Kwan MK, Myers RR, Brown RA, Triggs KJ, Woo SL and GarfinSR.An in vitro mechanical and histological study of acute stretching on rabbit tibial nerve. J Orthop Res. 1990;8:694-701.

23. Mahmud A, Ismail M, Álvaro RCM, Irênio G, Jefferson B, Daniel BN. Relationship between adverse neural tension and nerve conduction studies in patients with symptoms of the carpal tunnel syndrome. ArqNeuropsiquiatr. 2006;64(2-A):277-82.

24. Mackinnon SE. Pathophysiology of nerve compression. Hand Clin. 2002;18:231-341.

25. LundborgG,RydevikB. Effects of stretching the tibial nerve of the rabbit: a preliminary study of the intraneural circulation and the barrier function of the perineurium. J Bone Joint Surg Br. 1973;55(1):390-401. 
26. Brown R, Cynthia LB, Kerry KG, Jean-Michel B, Philip SS, Roger CJ, Michael PS. The effects of stretching upon conduction properties in the rabbit tibial nerve at the ankle: an unembalmed cadaveric study. J Man ManipTher. 2011;19 (1).

27. Dilley A, Lynn B, Pang SJ.Pressure and stretch mechanosensitivity of peripheral nerve fibres following local inflammation of the nerve trunk.Pain. 2005;117(3):462-72.

28. Peter JD, Michael AG, Graham ML. An in vivo study of peripheral nerves in continuity: biomechanical and physiological responses to elongation. J Orthop Res. 2002;20:370-5.

29. Wall EJ, Massie JB, Kwan MK. Experimental stretch neuropathy: changes in nerve conduction under tension. J Bone Joint Surg Br. 1992;74-B:126-9.

30. Take O,MatuzakiH, Hoshino M. Pathology and pressure of the nerve root at the herniated site during straight-leg-raising test: lumbar disc herniation. J JpnOrthopAssoc 1989;63:S1008.

31. Fleming P,LenehanB, O_Rourke S. Strain on the human sciatic nerve in vivo during movement of the hip and knee. J Bone Joint Surg Br. 2003;85B:363-5.

32. Coppieters MW, AlshamiAM. Longitudinal Excursion and Strain in the Median Nerve during Novel Nerve Gliding Exercises for Carpal Tunnel Syndrome. Journal of orthopaedicresearch : official publication of the Orthopaedic Research Society. 2007;25:97280 .

33. Schmidhammer R, ZandiehS,HopfR,MiznerI,PelinkaLE,KroepflA et al. Alleviated tension at the repair site enhances functional regeneration: the effect of full range of motion mobilization on the regeneration of peripheral nerves--histologic, electrophysiologic, and functional results in a rat model. J Trauma. 2004;56(3):571-84. Epub 2004/05/07.

34. RozmarynLM,Dovelle S, Rothman ER, Gorman K, OlveyKM,Bartko JJ. Nerve and tendon gliding exercises and the conservative management of carpal tunnel syndrome. Journal of hand therapy: official journal of the American Society of Hand Therapists. 1998;11(3):1719.

35. Myers RR, Murakami H, Powell HC. Reduced nerve blood flow in edematous neuropathies: a biomechanical mechanism.Microvasc Res. 1986;32(2):145-51.

36. Sunderland IRP, Brenner MJ, Singham J, Rickman RS, Hunter DA and Mackinnon SE. Effect of tension on nerve regeneration in rat sciatic nerve transection model. Ann Plast Surg. 2004; 53(4):382-7.

37. Boyd SB, Puttlitz C, GanJ,Topp KS. Strain and excursion in the rat sciatic nerve during a modified straight leg raise are altered after traumatic nerve injury. Journal of orthopaedicresearch : official publication of the Orthopaedic Research Society. 2005;23:76470. 
38. Singh A, Kallakuti S, Chen C, and Cavanaugh JM. Abstract. Structural and Functional Changes in Nerve Roots Due to Tension at Various Strains and Strain Rates: An In-Vivo Study. J Neurotrauma2009;26:627-40.

39. Ogata K, Naito M. Blood flow of peripheral nerve effects of dissection, stretching and compression. J Hand Surg Br 1985;11B:10-4.

40. Richard FE, Wayne AH, Neural Mobilization: A Systematic Review of Randomized Controlled Trials with an Analysis of Therapeutic Efficacy. J Man ManipTher.2009; 16: 8-22. 